\title{
Cross-sectional characterization of the dewetting of a Au/Ni Bilayer Film
}

\author{
Xi Cen, Andrew Thron, Xinming Zhang, Klaus van Benthem \\ Department of Chemical Engineering and Materials Science, University of California, Davis, CA 95616
}

\begin{abstract}
The solid state dewetting of $\mathrm{Au} / \mathrm{Ni}$ bilayer films was investigated by cross-sectional transmission electron microscopy techniques, including energy-dispersive X-ray spectroscopy, electron energy-loss spectroscopy and precession electron diffraction. After annealing under high vacuum conditions the early stage of film agglomeration revealed significant changes in film morphology and chemical distribution. Both $\mathrm{Au}$ and $\mathrm{Ni}$ showed texturing. Despite the initial deposition sequence of the as-deposited $\mathrm{Au} / \mathrm{Ni} / \mathrm{SiO}_{2} / \mathrm{Si}$ interface structure, the majority of the metal $/ \mathrm{SiO}_{2}$ interface was $\mathrm{Au} / \mathrm{SiO}_{2}$ after annealing at $675^{\circ} \mathrm{C}$ for 1 hour. Void nucleation was predominantly observed at $\mathrm{Au} / \mathrm{Ni} / \mathrm{SiO}_{2}$ triple junctions, rather than grain boundary grooving at free surface of the metal film. Detailed cross-sectional characterization reveals that the Au/Ni interface in addition to small amounts of metal alloying strongly affects film break-up and agglomeration kinetics. The formation of $\mathrm{Au} / \mathrm{SiO}_{2}$ interface sections is found to be energetically preferred over $\mathrm{Ni} / \mathrm{SiO}_{2}$ due to compressive stress in the as-deposited $\mathrm{Ni}$ layer. Void nucleation is observed at the film/substrate interface, while the formation of voids at $\mathrm{Ni} / \mathrm{Au}$ phase boundaries inside the metal film is caused by the Kirkendall effect.
\end{abstract}

Keywords: Gold/nickel bilayer film; Dewetting; Void formation 


\section{Introduction}

Solid state dewetting is a result of an imbalance between interface and surface free energies for a thin film on a solid substrate [1,2]. Thin film agglomeration can be a serious inter-circuit breaking issue in microsystem fabrication [3], while the mechanisms can also be used for preparing self-assembled arrays of catalyst for the growth of nanotubes and nanowires [4-6]. Hence, a comprehensive understanding of the mechanisms of solid-state dewetting is important for controlling processing steps in the fabrication of microelectronics and arrays of self-assembled catalyst sites. Agglomeration of polycrystalline films can be initiated by grain boundary grooving where the grain boundary intersects the free surface of the film, or by the formation of voids at the film/substrate interface [7, 8]. Once a hole in the film has formed, it will grow subsequently by continuous diffusion exposing the free surface of the underlying substrates [9]. Wetting-dewetting transitions have been extensively studied in single component films, but only few studies are reported for multi-component films [10-18].

Alloying and interdiffusion have been shown to affect the wetting and dewetting transitions in multi-component and bilayered films. Alloying of Au films with small amounts of Pt was observed to delay the onset of dewetting, while the underlying kinetics that caused the delay were not experimentally confirmed [10]. The addition of Ag into $\mathrm{Ni}$ films was shown to induce a fractal-like growth mode of voids rather than the expected capillarity driven hole nucleation and growth initiated by grain boundary grooving [11]. Dewetting of Ni-Cr alloyed films showed that different annealing conditions resulted in a variety of dewetting behaviors due to surface oxidation and film-substrate interaction, which were affected by different oxygen affinity of the two metals [12]. Single crystal 
$\mathrm{Au} / \mathrm{Fe}$ and $\mathrm{Fe} / \mathrm{Pd}$ bilayer films showed enhanced thermal stability upon annealing due to alloying effects $[13,14]$.

The Au/Ni bilayer model system used in this work, shows a homogeneous solid solution for temperatures above $816^{\circ} \mathrm{C}$, and a varying miscibility below $816^{\circ} \mathrm{C}$ [15]. Early studies on Au/Ni bilayer dewetting in Ar ambient revealed that the morphology of dewetted islands varies with annealing temperatures due to variations in the solubility limits [16-18]. Alloying starts to delay the hole formation above $700^{\circ} \mathrm{C}$ due to the formation of an intermediate phase [17]. Supersaturated, submicron-size Au-Ni islands can form when a Ni/Au bilayer film is annealed at temperatures above the miscibility gap, and subsequently quenched [18]. Phase separation from supersaturated Au/Ni particles was investigated by cross-sectional scanning electron microscopy (SEM) after additional annealing at $650^{\circ} \mathrm{C}[19]$. It was found that at equilibrium, the Ni-rich phases formed $<100>$ facets, rather than the $\langle 111\rangle$ facets. A preference toward the $\langle 100\rangle$ facets was attributed to a minimization of elastic energy at the interface between the $\mathrm{Ni}$ and $\mathrm{Au}$ rich phases. The studies reviewed above were mostly focused on how the amount of alloying affects the agglomeration behavior of $\mathrm{Ni} / \mathrm{Au}$ bilayer films. Interface configurations during the early stages of bilayer film agglomeration are not reported in the literature, but are critical for a detailed understanding of the break-up behavior of multi-component thin films. Void nucleation and thermal grain boundary grooving were previously discovered by cross-sectional SEM imaging [17]. More detailed transmission electron microscopy (TEM) characterization is, however, required to interrogate the changes in structural morphologies and chemical distributions. 
In this study we report changes in chemistry and microstructure of Au/Ni bilayer film during isothermal annealing, and the effects these changes have on the agglomeration of the bilayer film. Changes in the morphology and microstructure were observed by crosssectional transmission electron microscopy (TEM) techniques, including scanning transmission electron microscopy (STEM), precession electron diffraction (PED), electron energy-loss spectroscopy (EELS) and energy-dispersive X-ray spectroscopy (EDXS). The experimental results reveal dominant void nucleation at $\mathrm{Au} / \mathrm{Ni}$ interfaces within the film and at the bilayer/substrate interface, strong texturing of both metals as a function of diffusivity, and rearrangement of phase separated $\mathrm{Au}$ and $\mathrm{Ni}$ grains strongly affected by the compressive stress state in the as-deposited metal films and different diffusivities between two elements.

\section{Experimental Details}

Nickel and gold film were sequentially deposited onto Si substrates each with a nominal thickness of $30 \mathrm{~nm}$. Prior to metal film deposition a $12 \mathrm{~nm} \mathrm{SiO} 2$ thick layer was thermally grown on the (100) surface of silicon substrates to avoid silicide formation [20]. Ni films were deposited at room temperature by DC magnetron sputtering at a power of $100 \mathrm{~W}$, a base pressure of $1.7 \times 10^{-8}$ torr, and an Ar atmosphere of 2 mtorr. The corresponding sputter rate was $1.4 \AA^{-1} s^{-1}$. Au films were subsequently deposited on top of $\mathrm{Ni}$ at room temperature, using a Denton vacuum desk II cold sputtering unit. Au sputtering was performed at a base pressure of 20 mtorr, and an Ar pressure of 75 mtorr. The sputtering current was set at $23 \mathrm{~mA}$ resulting in sputtering rates around $2.5 \AA^{-1}$. Prior to deposition the $\mathrm{SiO}_{2} / \mathrm{Si}$ substrates were cleaned by sonication in methanol and deionized water immediately before insertion into the deposition chamber. 
The as grown samples were annealed in a three-zone tube furnace (Lindberg) at a base pressure of $6 \times 10^{-6}$ torr at $675^{\circ} \mathrm{C}$ for 1 hour. To avoid contamination the samples were placed in an alumina boat during annealing. Fast heating and cooling were achieved by pushing and withdrawing the quartz tube containing the samples into and out of the hot zone of the furnace [21].

Cross-sectional TEM specimens were prepared by standard FIB lift-out techniques $[22,23]$ using a FEI Scios dual-beam instrument. Film morphology and microstructures were characterized using a JEOL JEM-2500SE transmission electron microscope. STEM annular dark field (ADF) imaging was carried out with a FEI Titan G2 80-200 at FEI Company (Hillsboro, OR) and an aberration corrected JEOL JEM 2100F/Cs scanning transmission electron microscope at UC Davis. All microscopes were operated at $200 \mathrm{kV}$. TEM specimens were imaged in an edge-on orientation, i.e., the $<110>$ direction of the Si substrate was parallel to the incoming electron beam.

EDXS elemental distribution maps were acquired using the FEI Super-X EDX detector attached to a FEI Titan G2 80-200, which was installed at FEI Company. EELS line scans were acquired using a Gatan Tridiem spectrometer attached to the aberration corrected JEOL JEM 2100F/Cs instrument.

Crystalline orientation maps were used to study the crystalline orientation of $\mathrm{Ni}$ and $\mathrm{Au}$ grains along the interface. PED was performed using the JEOL JEM 2100F/Cs equipped with the NanoMEGAS Digistar hardware controlled by the Topspin ${ }^{\mathrm{TM}}$ precession control platform (AppFive LLC, Arizona). The step size was $1 \mathrm{~nm}$ with precession angle of $0.5^{\circ}$. Detailed information regarding the underlying physics of this technique are reported elsewhere [24-26]. 
The electron diffraction patterns were recorded from 25 different areas of one TEM sample. The width of the polycrystalline ring in each electron diffraction pattern was measured three times independently and calibrated using the simultaneously acquired silicon diffraction signal. The calculated lattice spacing was compared with tabulated values of bulk Ni to calculate strain. The stress was then determined based on the following equation: [27]

$$
\sigma=\frac{E}{1-v} \times \varepsilon
$$

$\mathrm{E}=200 \mathrm{GPa}$ is the elastic modulus of $\mathrm{Ni}$, and $v=0.3$ is the Poisson ratio.

\section{Results}

Fig. 1a shows a cross-sectional TEM bright field micrograph of the bilayer film before annealing. A difference in image contrast can be observed between the top and bottom layers of the bilayer film, with the Ni layer having a brighter contrast than the $\mathrm{Au}$ layer (Fig. 1a). An abrupt change in contrast at the $\mathrm{Ni} / \mathrm{Au}$ interface indicates no intermixing has occurred between Au and Ni before annealing. After annealing (Fig. 1b), re-arrangements of $\mathrm{Au}$ and $\mathrm{Ni}$ grains is observed throughout the metal film and separate $\mathrm{Au}$ and Ni layers can no longer be identified. Moreover, the film becomes semicontinuous, and the formation of holes and voids are observed. In some areas, indicated by an arrow, holes have formed in the annealed film and expose the underlying substrate. Slightly higher contrast at the substrate surface underneath the voids results from preferential thinning during FIB preparation.

The chemical distribution of the as-deposited film was further characterized with STEM-EELS line scans. Contrast in the ADF image in Fig. 2a shows the clear separation 
of $\mathrm{Au}$ and $\mathrm{Ni}$ at the top and bottom of the film, respectively. Furthermore, a thin $2 \mathrm{~nm}$ wide layer is observed to separate the Au and Ni layers (Fig. 2a). EELS line scans (Fig. 2b) acquired from the area observed in Fig 2a demonstrate an increase in the integrated oxygen $\mathrm{K}$ edge intensity at the $\mathrm{Ni} / \mathrm{Au}$ interface, confirming the presence of a roughly $2 \mathrm{~nm}$ wide native $\mathrm{NiO}$ layer at the $\mathrm{Ni} / \mathrm{Au}$ interface.

The chemical distribution of the annealed film was characterized with STEM-EDXS mapping. Fig. 3a shows a STEM HAADF survey image that was used during spectrum acquisition. Fig. $3 b$ and $3 c$ display corresponding EDXS chemical distributions maps obtained from spectral intensities of the $\mathrm{Ni} \mathrm{L}_{12}$ (yellow) and $\mathrm{Au} \mathrm{M} \alpha_{1}$ (purple), respectively. The same color codes are used for subsequent plots of spectral intensities throughout the remainder of this manuscript. The result confirms the direct correlation of dark field image intensities with the presence (or respective absence) of $\mathrm{Ni}$ and $\mathrm{Au}$, respectively. On the right side, as indicated by the red arrow in Fig 3a, both $\mathrm{Ni}$ and $\mathrm{Au}$ intensity co-exist in the EDXS mapping, which is likely to result from grain overlapping due to relatively smaller grain size.

In the region of interest, $\mathrm{Au} / \mathrm{SiO}_{2}$ occupies most of the interface with $\mathrm{Ni}$ grains sitting on top. A TEM analysis of a $10 \mu \mathrm{m}$ long section of the interface complemented by the characterization of 2 cross-sectional SEM samples revealed that roughly $54 \%$ of the film/substrate interface is comprised of $\mathrm{Au} / \mathrm{SiO}_{2}$, while only $21 \%$ shows a $\mathrm{Ni} / \mathrm{SiO}_{2}$ configuration. The remaining $25 \%$ of the substrate surface is exposed by either holes or voids in the film.

PED was used to acquire crystallographic information from the same area as Fig. 3. Phase maps were created by matching the acquired electron diffraction patterns with 
calculated templates for $\mathrm{Ni}$ and $\mathrm{Au}$ at various different orientations. Phase maps were created using the Topspin software to determine the crystalline phase and orientation in each area of the film. In Fig. 4a, red areas in the phase map correspond to the Ni FCC phase, while yellow areas correspond to Au FCC phase. The derived phase map is in excellent agreement with the EDX composition maps, except at positions where the $\mathrm{Au}$ and Ni grains overlap. An orientation map was created using the PED patterns and is shown in Fig. 4b. The image contrast in Fig. 4b represents the crystallographic direction of the film, normal to the film/substrate interface. Each color corresponds to the directions observed in the inverse pole figure to the right of Fig. $4 \mathrm{~b}$. Within one phase, several colors were observed indicating multiple grains. Stacked Ni and Au grains, as marked in Fig. 4b, stay in the similar crystalline orientation around $\langle 853\rangle$.

Texturing of the two metals in the annealed film was determined from the PED data using the harmonic extension method [28]. Fig. 5a and 5b show texture intensity plots along the interface normal, for $\mathrm{Au}$ and $\mathrm{Ni}$ grains, respectively. The presented inverse pole figures are considered representative as they were calculated from PED data obtained from a $6.5 \mu \mathrm{m}$ long interface section including around 640 individual grains. Intensities in these inverse pole figures scale with the observed frequency of a given crystallographic orientation. Both $\mathrm{Au}$ and $\mathrm{Ni}$ show strong texture along the direction normal to the interface, with Au predominantly oriented along the $\langle 111\rangle$ direction. Conversely Ni grains show texture around the $\langle 213>$ direction with a frequency $50 \%$ less than that for $\mathrm{Au}$ along the $<111>$ direction. $\mathrm{Ni}$ is also observed to have some texture around the $\langle 111\rangle$ and $\langle 001\rangle$ directions. However, these orientations are $\sim 33 \%$ less frequent than the $<213>$ direction. 
Figs. 6a and 6b show an ADF STEM and a bright field TEM micrograph of an interface between a $\mathrm{Ni}$ and $\mathrm{Au}$ grain after a void has formed at the $\mathrm{Ni} / \mathrm{Au} / \mathrm{SiO}_{2}$ triple junction. Due to the difference in atomic number $\mathrm{Ni}$ and $\mathrm{Au}$ grains can clearly be distinguished from each other (cf. Fig. 6a). The bright field TEM image in Fig. 6b indicates a rather well-defined $\mathrm{Au}$ surface at the void, while the Ni surface has a more diffuse contrast.

EELS line profiles were acquired across the Ni grain from the area marked in Fig. 6a in the direction normal to the interface plane. Integrated spectral intensities for the $\mathrm{Ni} \mathrm{L}_{2,3}$ and oxygen $\mathrm{K}$ edges are plotted as a function of relative distance in Fig. 7a. At the top surface of the $\mathrm{Ni}$ grain both the $\mathrm{Ni} \mathrm{L}_{2,3}$ and oxygen $\mathrm{K}$ edge intensities begin to increase. Inside the $\mathrm{Ni}$ grain, the oxygen $\mathrm{K}$-edge signal diminishes while the $\mathrm{Ni}$ signal continuously increases. Approaching the void the integrated oxygen K-edge signal reveals a strong signal with a full-width at half maximum (FWHM) around 8-10 nm. Inside the void both $\mathrm{O}$ and $\mathrm{Ni}$ signals become indistinguishable from noise. Si L2,3 edge was also detected at the bottom surface of the Ni grain as marked in Fig. 6a. Fig. $7 \mathrm{~b}$ shows the background subtracted fine structure of the $\mathrm{Si} \mathrm{L}_{2,3}$ edge acquired from the bottom surface, which is in excellent agreement with the near edge fine structure found in reference spectra for bulk $\mathrm{SiO}_{2}$ [29]. From the results in Fig. 7 it is concluded that $\mathrm{SiO}_{2}$ is present at the bottom surface of the Ni grain, adjacent to the void.

Void formation was also observed at $\mathrm{Ni} / \mathrm{Au}$ phase boundaries inside the metal film. Fig. 8a shows a HAADF STEM survey image of an area where a void formed at the $\mathrm{Ni} / \mathrm{Au}$ grain boundary. Figs. $8 \mathrm{~b}$ and 8c display EDXS chemical distributions maps for $\mathrm{Au}$ and $\mathrm{Ni}$, respectively. In Fig $8 \mathrm{a}$, the nanostructure displays inclined grain boundaries 
between $\mathrm{Ni}$ and $\mathrm{Au}$, which divide the region of interest into three areas. Area 1 towards the left is comprised predominantly of $\mathrm{Au}$, while area 3 on the right side of Fig. 8a is primarily composed of $\mathrm{Ni}$. The central area, area 2, represents an overlap of $\mathrm{Au}$ and $\mathrm{Ni}$ rich phases along the viewing direction. In area 2, a region with darker contrast and a faceted shape is observed. The chemical distribution map for Au (Fig. 8b) reveals an absence of intensity in the same region, while the Ni distribution map shows only a slight decrease in intensity (Fig. 8c). These observations suggest that the faceted void is predominantly located inside the Au rich grain that may also extent slightly into the $\mathrm{Ni}$ rich grain.

The shape of the void shows mostly faceting of the adjacent metal phases, which is clearly observed by HRTEM (Fig. 9a). Some edges of the void reveal, however, slightly rounded shape. The lattice contrast observed inside the void originates from the Ni rich phase located in the same field of view due to the projection mode for TEM imaging. Moiré contrasts surrounding the void confirm that $\mathrm{Au}$ and Ni grains overlap along the viewing direction, while the absence of Moire patterns is expected from a void which is directly in front of or behind the Ni rich grain. The void shape was calculated using the Wulffmaker software [30], based on the assumption that the void obeys the same Wulff shape as Au particles. The orientation of Au crystal was obtained from the precession assisted diffraction patterns. The free surface energy of (100), (110), and (111) planes for Au were extracted from references [35] (see Table 1) and used for modeling. Overall the projected outline of the calculated void shape (Fig. 9b) is overlaid on the HRTEM micrograph (Fig. 9a) and agrees surprisingly well with the experimentally observed geometric shape. 


\section{Discussion}

\subsection{Film morphology at the early stages of dewetting}

In the as-deposited metal bilayer film, the $\mathrm{Ni}$ and $\mathrm{Au}$ layers are polycrystalline, continuous and uniform in thickness. Cross-sectional EELS line scans reveal a 2-3 nm continuous native $\mathrm{NiO}_{\mathrm{x}}$ layer formed between $\mathrm{Ni}$ and $\mathrm{Au}$, which formed as a surface oxide on the top of the Ni film prior to Au deposition. The oxide layer likely obstructs inter-diffusion of $\mathrm{Au}$ and $\mathrm{Ni}$ at low temperatures while cross-sectional TEM and STEM imaging reveals that at elevated temperatures inter-diffusion eventually occurs. Figs 3 and 4 demonstrate that in some areas Au has diffused underneath the Ni film to replace the $\mathrm{Ni} / \mathrm{SiO}_{2}$ interface by the $\mathrm{Au} / \mathrm{SiO}_{2}$ interface. Similar observations were reported previously for the annealing of $5 \mathrm{~nm}$ thick Au films on top of $10 \mathrm{~nm}$ Ni films in air [31]. Chen et al. have argued that the driving force in this case was the difference of oxygen affinity between $\mathrm{Ni}$ and $\mathrm{Au}$. In this study, however, annealing was carried out in vacuum, i.e., at an $\mathrm{O}_{2}$ partial pressure of $7 \times 10^{-8}$ torr. The interface energies for $\mathrm{Au} / \mathrm{SiO}_{2}$ and $\mathrm{Ni} / \mathrm{SiO}_{2}$ can be estimated by the following equation [32]:

$$
\gamma_{\text {film/substrate }}=\gamma_{\text {film/vapor }}+\gamma_{\text {substrate/vapor }}-W_{\text {adhesive }} .
$$

The work of adhesion $W_{\text {adhesive }}$ for $\mathrm{Ni}$ and $\mathrm{Au}$ on $\mathrm{SiO}_{2}$, and the surface energies $\gamma$ for $\mathrm{Ni}$, $\mathrm{Au}$ and $\mathrm{SiO}_{2}$ were taken from references [33-38] and are listed in Table 1. From the reference values, interface energies were calculated for $\mathrm{Au} / \mathrm{SiO}_{2}$ and $\mathrm{Ni} / \mathrm{SiO}_{2}$ configurations, and listed in Table 1. Due to the anisotropic nature of Au and Ni surface energies, the calculated interface energies strongly depend on the orientation of the respective metal film. The difference of interface energy between two different types of interface is within $0.3 \mathrm{~J} / \mathrm{m}^{2}$, which is similar to the difference between calculated [35] and 
experimentally determined free surface energies values [36, 37] (see Table 1). In addition, it must be noted that the values for the work of adhesion extracted from reference 34 were determined by fracture measurements that include irreversible processes such as plastic deformation [39]. Hence, the $\mathrm{Au} / \mathrm{SiO}_{2}$ or $\mathrm{Ni} / \mathrm{SiO}_{2}$ interface energies listed in Table 1 do not provide conclusive evidence for which interface configuration is energetically favorable.

The PED analysis of annealed bilayer films has demonstrated that $\mathrm{Au}$ grains are highly textured around $\langle 111\rangle$, while Ni grains reveal $\langle 213\rangle$ orientation parallel to the substrate's interface normal. The (213) free surface energy for $\mathrm{Ni}$ is higher than the lower-index Ni surface energies listed in Table 1 [40]. According to equation (2) Ni (213) planes in contact with the underlying $\mathrm{SiO}_{2}$ therefore have a higher interface energy compared to lower-indexed $\mathrm{Ni} / \mathrm{SiO}_{2}$ interfaces. Analysis of electron diffraction patterns furthermore revealed that the polycrystalline as-deposited Ni film was under $0.79 \pm 0.17$ GPa compressive stress caused by the sputter deposition process at room temperature. Under this circumstance the as-deposited $\mathrm{Ni} / \mathrm{SiO}_{2}$ interface energy is considered even higher than suggested by Table 1 [41]. Therefore, it is concluded that the driving force for $\mathrm{Au}$ diffusion underneath the previously continuous $\mathrm{Ni}$ film is the lowering of the metal $/ \mathrm{SiO}_{2}$ interface energy. The formation of the $\mathrm{Au} / \mathrm{SiO}_{2}$ interface contributes in delaying thin film agglomeration of the bilayer film, in conjunction with the previously observed alloying effects.

Ni grains are also expected to obtain a $<111>$ texture similar to Au. Kinetics, however, have a significant effect on the rearrangement of $\mathrm{Ni}$ and $\mathrm{Au}$ at the early stage of film agglomeration. Free surface diffusion of $\mathrm{Ni}$ was suppressed by both the $\mathrm{NiO}$ and $\mathrm{Au}$ 
layers, and, therefore, obstructed grain boundary grooving. In addition, the diffusivity of $\mathrm{Au}$ is significantly larger than that of $\mathrm{Ni}$ [42], which promotes Au migration as the kinetically dominant mechanism to facilitate the minimization of the metal/substrate interface energy.

The ADF-STEM micrograph in Fig. 10 shows a boundary between two adjacent Ni grains. Under the respective imaging conditions, bright intensities represent $\mathrm{Au}$, while lower intensities signify Ni. The grain boundary exhibits a sharp bright image intensity, indicating that $\mathrm{Au}$ diffused along the grain boundary during annealing, forming a $\mathrm{Au}$ rich grain underneath the two Ni grains. The diffuse bright intensity in the Ni grains, near the grain boundary, may also indicate residual Au-Ni alloying that occurred at elevated temperatures. As the temperature increases, the contribution of bulk self-diffusion increases [43], as well as the amount of Au-Ni alloying. Hence, Au may also diffuse through $\mathrm{Ni}$ grains towards the substrate surface and form an $\mathrm{Au} / \mathrm{SiO}_{2}$ interface. Both mechanisms lead to the formation of the observed $\mathrm{Au} / \mathrm{SiO}_{2}$ interface driven by the reduction of total interface energy. The observed sub-stoichiometric nickel oxide layer between the as-deposited metal layers revealed no significant influence on the morphological changes of the metal film upon thermal annealing.

Electron diffraction pattern analysis has revealed that the polycrystalline Ni film was under compressive stress due to sputter deposition. It is, therefore, concluded that the $\mathrm{Au} / \mathrm{SiO}_{2}$ interface energy is lower than that for a $\mathrm{Ni} / \mathrm{SiO}_{2}$ interface configuration given that the Ni film is under compressive stress [41]. The experimental observations suggest that $\mathrm{Au}$ diffusion outruns relaxation of the $\mathrm{Ni}$ film and, therefore, forms a predominant $\mathrm{Au} / \mathrm{SiO}_{2}$ interface. Surprisingly, no remaining $\mathrm{Au}$ absorption layers on top of 
agglomerated $\mathrm{Ni}$ grains were detected by either EDXS elemental mapping or atomic resolution HAADF-STEM imaging.

\subsection{Void nucleation}

Voids were predominantly observed at $\mathrm{Ni} / \mathrm{Au} / \mathrm{SiO}_{2}$ triple junctions. Void formation at the $\mathrm{Ni} / \mathrm{SiO}_{2}$ interface during thermal annealing was previously observed [44], and the compressive stress of the as-deposited Ni layer may have caused delamination through buckling [45]. HRTEM imaging combined with EELS characterization has revealed a $\mathrm{NiO}$ layer at the $\mathrm{Ni}$ /void interface in Fig 6, while some $\mathrm{SiO}_{2}$ was also detected in the same area. The same EELS characterization was conducted for the Au grain on the opposite side of the phase boundary, but no $\mathrm{Si} \mathrm{L}_{2,3}$ signal was detected. These observations may indicate partial delamination, i.e., buckling, of the as-deposited Ni film due to compressive stress in addition to local void formation. The interfacial fracture energy for $\mathrm{Ni} / \mathrm{SiO}_{2}$ is higher than that for $\mathrm{Au} / \mathrm{SiO}_{2}\left(1.37 \mathrm{~J} / \mathrm{m}^{2}\right.$ vs. $0.39 \mathrm{~J} / \mathrm{m}^{2}$, respectively $[33,34])$, which is consistent with this interpretation.

The observation of voids predominantly at $\mathrm{Ni} / \mathrm{Au} / \mathrm{SiO}_{2}$ triple junctions suggests that voids were formed after the rearrangement of $\mathrm{Ni}$ and $\mathrm{Au}$. Evidence of void nucleation and grain boundary grooving for $\mathrm{Au} / \mathrm{Ni}$ bilayers was previously discovered by crosssectional SEM imaging [17]. In this cross-sectional TEM study, however, only the formation of voids was observed. During the rearrangement of $\mathrm{Ni}$ and $\mathrm{Au}$, the difference in the interdiffusion coefficient of $\mathrm{Au}$ and $\mathrm{Ni}$ could induce a high density of vacancies in the film. Moreover, alloying and the formation of $\mathrm{Au} / \mathrm{Ni}$ grain boundaries could also cause stress due to a large lattice mismatch of $15 \%$ [46]. As a consequence, void nucleation was found to be the dominant mechanism of hole formation during the early 
stages of the dewetting process. It must be noted that the top Au layer may have started to dewet early during annealing due to its higher diffusivity and large surface area. More extensive in-situ TEM studies would be required to provide additional evidence, but is beyond the scope of this publication.

Besides the void nucleation at $\mathrm{Ni} / \mathrm{Au} / \mathrm{SiO}_{2}$ triple junction, Kirkendall void formation was also observed at $\mathrm{Ni} / \mathrm{Au}$ phase boundaries. According to the phase diagram, intermixing of $21 \% \mathrm{Ni}$ in $\mathrm{Au}$, and $9 \% \mathrm{Au}$ in $\mathrm{Ni}$ occurs at $675^{\circ} \mathrm{C}$. The diffusivity of $\mathrm{Au}$ in $\mathrm{Ni}$ is, however, higher than that for Ni in Au. Thus, the Kirkendall effect [47] causes void formation at the phase boundary but within the Au phase. In this study, voids around $\mathrm{Au} / \mathrm{Ni}$ grain boundaries were located to be predominantly within the Au phase (cf. Fig. 8). Kirkendall voids likely act as a source of vacancies during dewetting, aiding in the formation of voids which are caused by capillary instabilities at metal/grain boundary/substrate triple junctions [48, 49]. Furthermore, Kirkendall voids create $\mathrm{Au} / \mathrm{Ni} / \mathrm{Void}$ triple junctions, where new voids can begin to nucleate. To our knowledge, Kirkendall voids have not been previously observed during the dewetting of bilayer films.

Faceting of the void inside the Au phase was observed due to the anisotropic surface energy of solid Au [36]. The surprising agreement of the Wulff constructed shape of the void with the experimental observations (cf. Fig 9a) indicates that the void and Au grains are approaching thermodynamic equilibrium. Observed differences between experimentally observed and modeled void shapes must origin from the intersecting $\mathrm{Au} / \mathrm{Ni}$ grain boundary, and the fact that thermodynamic equilibrium has not yet been reached. The rather complicated morphology of the annealed bilayer film indicates, however, that the agglomeration process to form homogeneous interface structures that 
correspond to the lowest energy configuration is hindered due to local intermixing of $\mathrm{Ni}$ and $\mathrm{Au}$ at elevated temperatures.

\section{Conclusion}

The agglomeration of $\mathrm{Au} / \mathrm{Ni}$ bilayer films on $\mathrm{SiO}_{2} / \mathrm{Si}$ substrates was studied in detail by cross-sectional TEM techniques. The observations revealed considerable changes in film morphology, chemical distribution, formation of texture, and void nucleation at triple points and $\mathrm{Au} / \mathrm{Ni}$ phase boundaries. Au diffused underneath the $\mathrm{Ni}$ film during the annealing of the as-deposited bilayer film, reduce the metal/ $\mathrm{SiO}_{2}$ interface energy by relieving the considerable compressive stress in the Ni film. The Au grains were able to achieve a $<111>$ texture due to the high mobility of Au, forming a more energetically favorable metal/ $\mathrm{SiO}_{2}$ interface compared with the higher indexed $\mathrm{Ni}$ grains. Formation of the $\mathrm{Au} / \mathrm{SiO}_{2}$ interface delays the agglomeration of the bilayer film, in conjunction with the previously observed alloying effect. Void formation is observed to be the dominant mechanism for the nucleation of holes during the early stages of film agglomeration. Besides nucleation at film/substrate triple junctions, the Kirkendall effect was observed to cause the formation of voids at $\mathrm{Au} / \mathrm{Ni}$ phase boundaries, with voids forming inside the Au phase. The formation of Kirkendall voids is another consequence of Au-Ni alloying which affects the agglomeration process in Au/Ni bilayer films.

\section{Acknowledgements}

This study was financially supported by a Faculty Early Career award from the US National Science Foundation (DMR-0955638). X.Z. was supported by UC Laboratory Fee grant \#12-LR-238313. We acknowledge Dr. Huikai Cheng and Dr. Liang Zhang at 
the FEI Nanoport for invaluable help during EDXS experiments. We acknowledge Dr.

Peter Greene and Dr. Kai Liu for invaluable help during thin film deposition.

\section{Reference}

[1] W.D. Kaplan, D. Chatain, P. Wynblatt, W.C. Carter. A review of wetting versus adsorption, complexions, and related phenomena: the rosetta stone of wetting, J Mater Sci 48 (2013) 5681-5717.

[2] J.W. Cahn, J.E. Hilliard. Free Energy of a Nonuniform System .1. Interfacial Free Energy, J Chem Phys 28 (1958) 258-267.

[3] D. Deduytsche, C. Detavernier, R.L. Van Meirhaeghe, C. Lavoie. High-temperature degradation of NiSi films: Agglomeration versus NiSi 2 nucleation, J Appl Phys 98 (2005).

[4] L. Kondic, J.A. Diez, P.D. Rack, Y.F. Guan, J.D. Fowlkes. Nanoparticle assembly via the dewetting of patterned thin metal lines: Understanding the instability mechanisms, Phys Rev E 79 (2009).

[5] J. Yun, R. Wang, W.K. Choi, J.T.L. Thong, C.V. Thompson, M. Zhu, Y.L. Foo, M.H. Hong. Field emission from a large area of vertically-aligned carbon nanofibers with nanoscale tips and controlled spatial geometry, Carbon 48 (2010) 1362-1368.

[6] J. Guan, B. Yu, L.J. Lee. Forming highly ordered arrays of functionalized polymer nanowires by dewetting on micropillars, Adv Mater 19 (2007) 1212-1214.

[7] W.W. Mullins. Theory of Thermal Grooving, J Appl Phys 28 (1957) 333-339.

[8] C.M. Kennefick, R. Raj. Copper on Sapphire - Stability of Thin-Films at 0.7-Tm, Acta Metallurgica 37 (1989) 2947-2952.

[9] C.V. Thompson. Solid-State Dewetting of Thin Films, Annu Rev Mater Res 42 (2012) 399434.

[10] C. Manuela Müller, R. Spolenak. Dewetting of Au and AuPt alloy films: A dewetting zone model, J Appl Phys 113 (2013) 094301.

[11] J. Petersen, S.G. Mayr. Dewetting of $\mathrm{Ni}$ and NiAg solid thin films and formation of nanowires on ripple patterned substrates, J Appl Phys 103 (2008) 023520.

[12] Y. Kwon, N.H. Kim, G.P. Choi, W.S. Lee, Y.J. Seo, J.S. Park. Structural and surface properties of NiCr thin films prepared by DC magnetron sputtering under variation of annealing conditions, Microelectron Eng 82 (2005) 314-320.

[13] D. Amram, L. Klinger, E. Rabkin. Anisotropic hole growth during solid-state dewetting of single-crystal Au-Fe thin films, Acta Mater 60 (2012) 3047-3056. 
[14] M. Perzanowski, Y. Zabila, J. Morgiel, A. Polit, M. Krupinski, A. Dobrowolska, M. Marszalek. AFM, XRD and HRTEM Studies of Annealed FePd Thin Films, Acta Phys Pol A 117 (2010) 423-426.

[15] J.H. Wang, X.G. Lu, B. Sundman, X.P. Su. Thermodynamic assessment of the Au-Ni system, Calphad 29 (2005) 263-268.

[16] D. Wang, P. Schaaf. Ni-Au bi-metallic nanoparticles formed via dewetting, Mater Lett 70 (2012) 30-33.

[17] A. Herz, D. Wang, T. Kups, P. Schaaf. Solid-state dewetting of Au/Ni bilayers: The effect of alloying on morphology evolution, J Appl Phys 116 (2014).

[18] A. Herz, D. Wang, R. Muller, P. Schaaf. Formation of supersaturated Au-Ni nanoparticles via.dewetting of an Au/Ni bilayer, Mater Lett 102 (2013) 22-25.

[19] A. Herz, M. Friak, D. Rossberg, M. Hentschel, F. Theska, D. Wang, D. Holec, M. Sob, O. Schneeweiss, P. Schaaf. Facet-controlled phase separation in supersaturated Au-Ni nanoparticles upon shape equilibration, Appl Phys Lett 107 (2015).

[20] A.M. Thron, P.K. Greene, K. Liu, K. van Benthem. Structural changes during the reaction of Ni thin films with (100) silicon substrates, Acta Mater 60 (2012) 2668-2678.

[21] D. Chatain, V. Ghetta, P. Wynblatt. Equilibrium shape of copper crystals grown on sapphire, Interface Sci 12 (2004) 7-18.

[22] M. Schaffer, B. Schaffer, Q. Ramasse. Sample preparation for atomic-resolution STEM at low voltages by FIB, Ultramicroscopy 114 (2012) 62-71.

[23] C.A. Volkert, A.M. Minor. Focused ion beam microscopy and micromachining, MRS Bulletin 32 (2007) 389-399.

[24] R. Vincent, P.A. Midgley. Double Conical Beam-Rocking System for Measurement of Integrated Electron-Diffraction Intensities, Ultramicroscopy 53 (1994) 271-282.

[25] J.G. Brons, G.B. Thompson. Orientation Mapping via Precession-Enhanced Electron Diffraction and Its Applications in Materials Science, Jom-Us 66 (2014) 165-170.

[26] A. Avilov, K. Kuligin, S. Nicolopoulos, M. Nickolskiy, K. Boulahya, J. Portillo, G. Lepeshov, B. Sobolev, J.P. Collette, N. Martin, A.C. Robins, P. Fischione. Precession technique and electron diffractometry as new tools for crystal structure analysis and chemical bonding determination, Ultramicroscopy 107 (2007) 431-444.

[27] M. Meyers, K. Chawla. Mechanical Behavior of materials. Cambridge University Press, New York, 2009.

[28] H.J. Bunge, Texture Analysis in Materials Science: Mathematical Methods. Butterworths, London, 1982 
[29] C.C. Ahn, P. Rez. Inner Shell Edge Profiles in Electron-Energy Loss Spectroscopy, Ultramicroscopy 17 (1985) 105-115.

[30] R.V. Zucker, D. Chatain, U. Dahmen, S. Hagege, W.C. Carter. New software tools for the calculation and display of isolated and attached interfacial-energy minimizing particle shapes, J Mater Sci 47 (2012) 8290-8302.

[31] L.C. Chen, F.R. Chen, J.J. Kai, L. Chang, J.K. Ho, C.S. Jong, C.C. Chiu, C.N. Huang, C.Y. Chen, K.K. Shih. Microstructural investigation of oxidized Ni/Au ohmic contact to p-type GaN, J Appl Phys 86 (1999) 3826-3832.

[32] M. Ohring, Materials Science of Thin Films, Second ed. Academic Press, Waltham, 2001

[33] M.S. Kennedy, N.R. Moody, D.P. Adams, M. Clift, D.F. Bahr. Environmental influence on interface interactions and adhesion of $\mathrm{Au} / \mathrm{SiO}_{2}$, Mat Sci Eng a-Struct 493 (2008) 299-304.

[34] C.H. Lee, J.H. Kim, C.Y. Zou, I.S. Cho, J.M. Weisse, W. Nemeth, Q. Wang, A.C.T. Van Duin, T.S. Kim, X.L. Zheng. Peel-and-Stick: Mechanism Study for Efficient Fabrication of Flexible/Transparent Thin-film Electronics, Sci Rep-Uk 3 (2013).

[35] E.A. Holm, D.L. Olmsted, S.M. Foiles. Comparing grain boundary energies in face-centered cubic metals: Al, Au, Cu and Ni, Scripta Mater 63 (2010) 905-908.

[36] H.P. Bonzel, M. Nowicki. Absolute surface free energies of perfect low-index orientations of metals and semiconductors, Physical Review B - Condensed Matter and Materials Physics 70 (2004) 1-9.

[37] H. Meltzman, D. Chatain, D. Avizemer, T.M. Besmann, W.D. Kaplan. The equilibrium crystal shape of nickel, Acta Materialia 59 (2011) 3473-3483.

[38] J. Bohm. Treatise on solid state chemistry. Plenum Press, New York 1975.

[39] J. Kim, K.S. Kim, Y.H. Kim. Mechanical effects in peel adhesion test, journal of Adhesion Science and Technology 3 (1989) 175-187.

[40] Y.N. Wen, H.M. Zhang. Surface energy calculation of the fcc metals by using the MAEAM, Solid State Commun 144 (2007) 163-167.

[41] T. Frolov and Y. Mishin, Thermodynamics of coherent interfaces under mechanical stress. I. Theory, Phys. Rev. B 85 (2012) 224106.

[42] M.J.H. van Dal, M.C.L.P. Pleumeekers, A.A. Kodentsov, F.J.J. van Loo. Diffusion studies and re-examination of the Kirkendall effect in the Au-Ni system, J Alloy Compd 309 (2000) 132-140.

[43] A. Kosinova, D. Wang, P. Schaaf, O. Kovalenko, L. Klinger, E. Rabkin. Fabrication of hollow gold nanoparticles by dewetting, dealloying and coarsening, Acta Mater 102 (2016) 108-115. 
[44] A.M. Thron, P. Greene, K. Liu, K. van Benthem. In-situ observation of equilibrium transitions in Ni films; agglomeration and impurity effects, Ultramicroscopy 137 (2014) 5565

[45] J.A. Thornton, D.W. Hoffman. Stress-Related Effects in Thin-Films, Thin Solid Films 171 (1989) 5-31.

[46] Y. Gao, K.L. Merkle. High-Resolution Electron-Microscopy of Metal Metal and Metal Metal-Oxide Interfaces in the Ag/Ni and Au/Ni Systems, J Mater Res 5 (1990) 1995-2003.

[47] H.J. Fan, M. Knez, R. Scholz, D. Hesse, K. Nielsch, M. Zacharias, U. Gosele. Influence of surface diffusion on the formation of hollow nanostructures induced by the Kirkendall effect: The basic concept, Nano Lett 7 (2007) 993-997.

[48] P.R. Gadkari, A.P. Warren, R.M. Todi, R.V. Petrova, K.R. Coffey. Comparison of the agglomeration behavior of thin metallic films on $\mathrm{SiO}_{2}$, J Vac Sci Technol A 23 (2005) 11521161.

[49] E. Shaffir, I. Riess, W.D. Kaplan. The mechanism of initial de-wetting and detachment of thin Au films on YSZ, Acta Mater 57 (2009) 248-256.

\section{Figure Captions}

Figure 1: Cross-sectional TEM bright field micrographs of the (a) as-deposited bilayer film and (b) the annealed bilayer film. The red arrow highlights the formation of a hole.

Figure 2: (a) An annular dark field image of as-deposited film. (b) The integrated EELS intensities of the oxygen $\mathrm{K}$ plotted as a function of relative position across bilayer film, from the arrow marker by the red arrow in (a)

Figure 3: EDXS chemical distribution maps and the corresponding STEM survey image. (a) The HAADF STEM survey micrograph. (b) and (c) are EDXS chemical distribution maps obtained from the spectral intensities of the $\mathrm{Ni}$ L $\alpha_{1 \_2}$ (yellow) and Au M $\alpha_{1}$ (purple), respectively. 
Figure 4: (a) Phase map calculated from PED. Red intensity signifies the Ni FCC phase while yellow intensities signify the Au FCC phase. (b) Orientation map for the direction normal to the interface with the color intensities corresponding to the inverse pole figure to the right of the image.

Figure 5: $\quad$ Texture intensity plot in the format of inverse pole figure of (a) Au and (b) $\mathrm{Ni}$, respectively.

Figure 6: (a) STEM and (b) bright field TEM micrographs of an interface between a $\mathrm{Ni}$ and $\mathrm{Au}$ grains, where a void has formed underneath the phase boundary.

Figure 7: (a) Plots of integrated EELS intensities for the Ni L2,3 and $\mathrm{O} \mathrm{K}$ edge as a function of relative position across the Ni grain, as marked in Fig. 6a by the red arrow. (b) $\mathrm{Si} \mathrm{L}_{2,3}$ edge recorded from the bottom area of $\mathrm{Ni}$ as marked by the red dot in Fig. 6a, plotted in comparison to a reference spectrum for bulk $\mathrm{SiO}_{2}[29]$.

Figure 8: $\quad$ EDXS chemical distribution maps and corresponding STEM survey image. (a) The HAADF STEM survey micrograph. (b) and (c) are EDXS chemical distribution maps obtained from spectral intensities of the $\mathrm{Au} \mathrm{M} \alpha_{1}$ (purple) and Ni L $\alpha_{1 \_2}$ (yellow), respectively. The dashed lines highlight areas where $\mathrm{Ni}$ and Au grains overlap. The dashed circle highlights a void observed in projection.

Figure 9: (a) The HRTEM micrograph of the void observed in Fig. 8. (b) The simulated void with the orientation obtained from the PED. The frame of facet planes are cropped from simulated void (b) and matched with the one in HRTEM micrograph, as shown by the dashed red lines in (a) 
Figure 10: Annular dark field micrograph showing the presence of $\mathrm{Au}$ at a $\mathrm{Ni}$ grain boundary, and the formation of a Au grain underneath the two Ni grains.

\section{Tables}

Table 1: Surface energy, adhesive energy and calculated interface energy for $\mathrm{Ni}$ and $\mathrm{Au}$ with $\mathrm{SiO}_{2}$. All values in $\mathrm{J} / \mathrm{m}^{2}$

\begin{tabular}{lll}
\hline Energy & $\mathrm{Au}$ & $\mathrm{Ni}$ \\
\hline Adhesive energy w $\mathrm{SiO}_{2}$ & $0.39^{[33]}$ & $1.37^{[34]}$ \\
Surface energy(cal) $(100)^{[35]}$ & 1.176 & 2.06 \\
Surface energy(cal) $(110)^{[35]}$ & 1.282 & 2.375 \\
Surface energy(cal) $(111)^{[35]}$ & 1.094 & 1.953 \\
Surface energy(exp) $(111)$ & $1.46^{[36]}$ & $2.05^{[37]}$ \\
Interface energy (100) $\mathrm{w} \mathrm{SiO}_{2}$ & 1.093 & 0.997 \\
Interface energy (110) $\mathrm{w} \mathrm{SiO} 2$ & 1.199 & 0.89 \\
Interface energy (111) $\mathrm{wiO}_{2}$ & 1.011 & \\
\hline SiO 2 free surface energy: $0.307^{[38]}$ & & \\
\hline
\end{tabular}




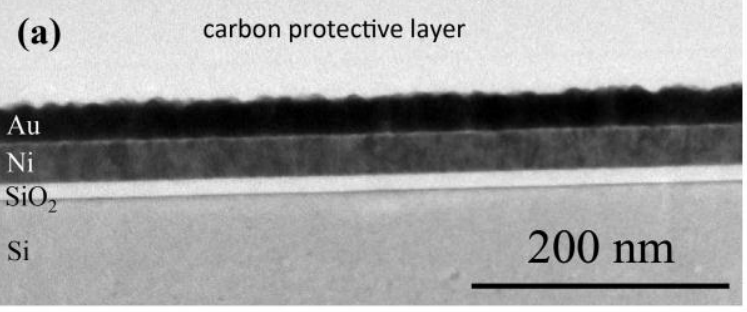

(b)

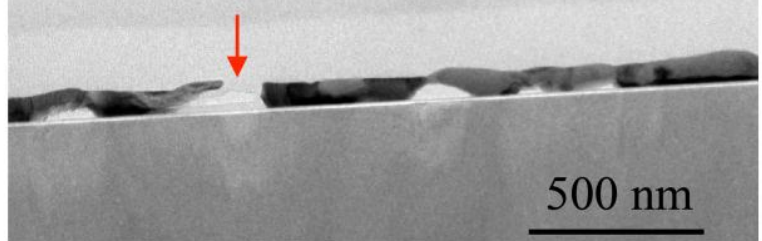


(a)

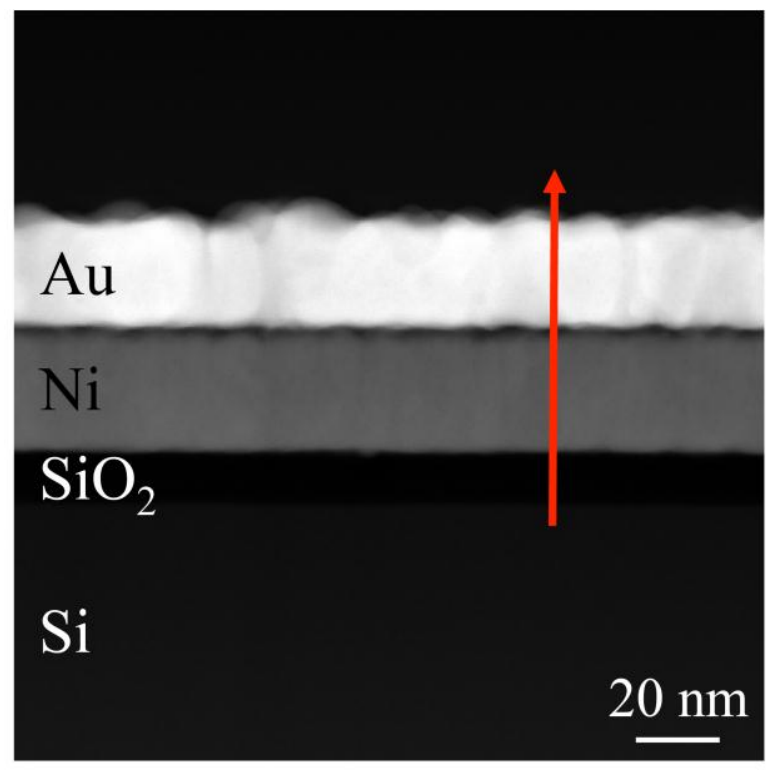

(b)

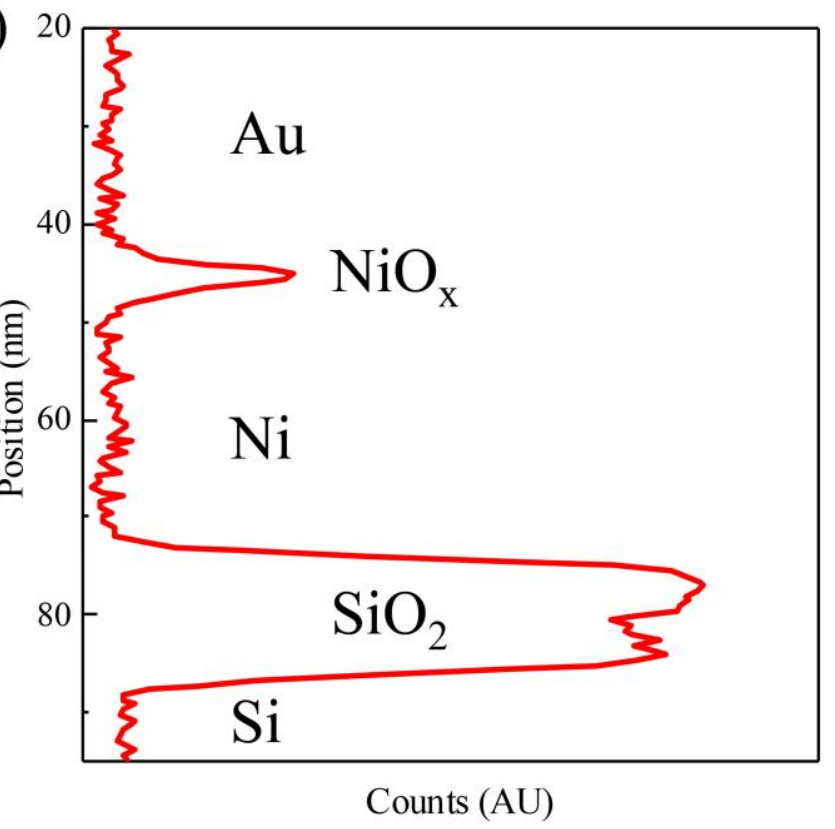




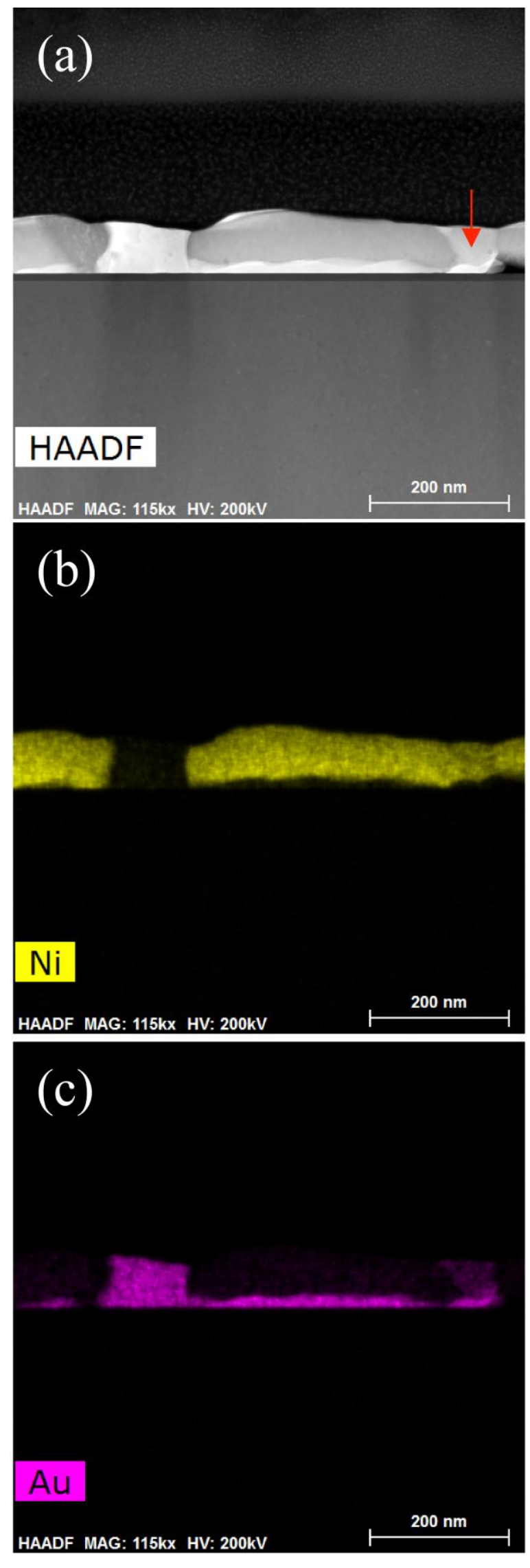



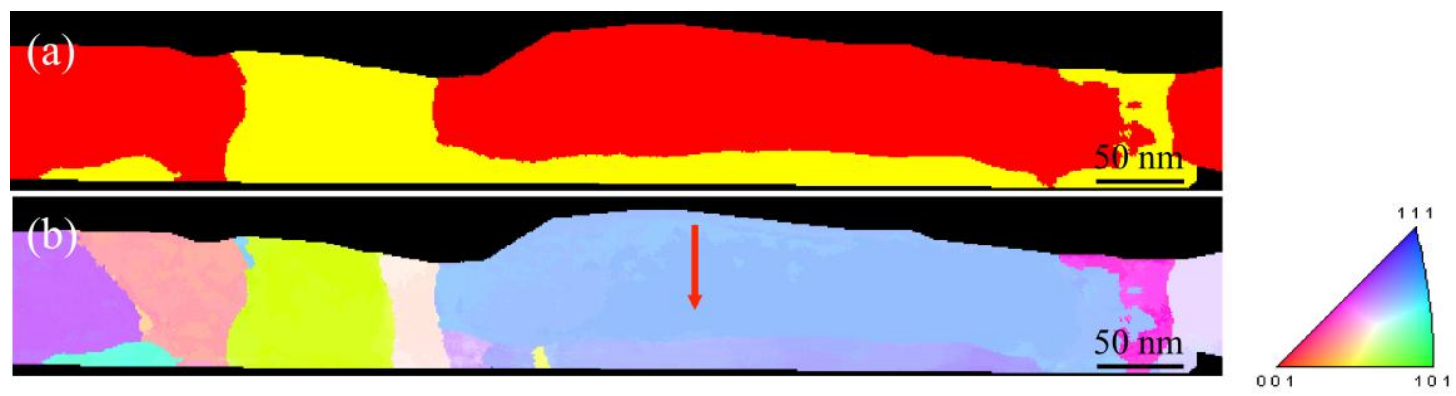
(a) Au texture

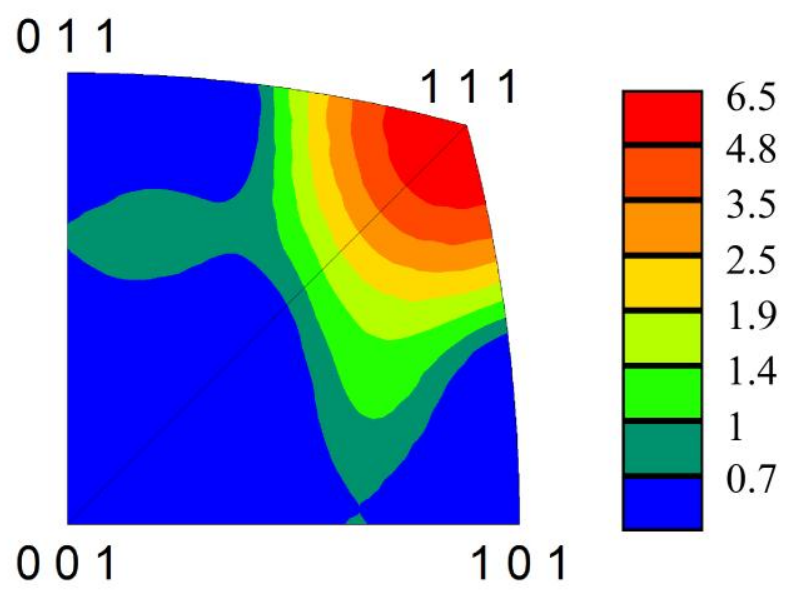

(b) Ni texture

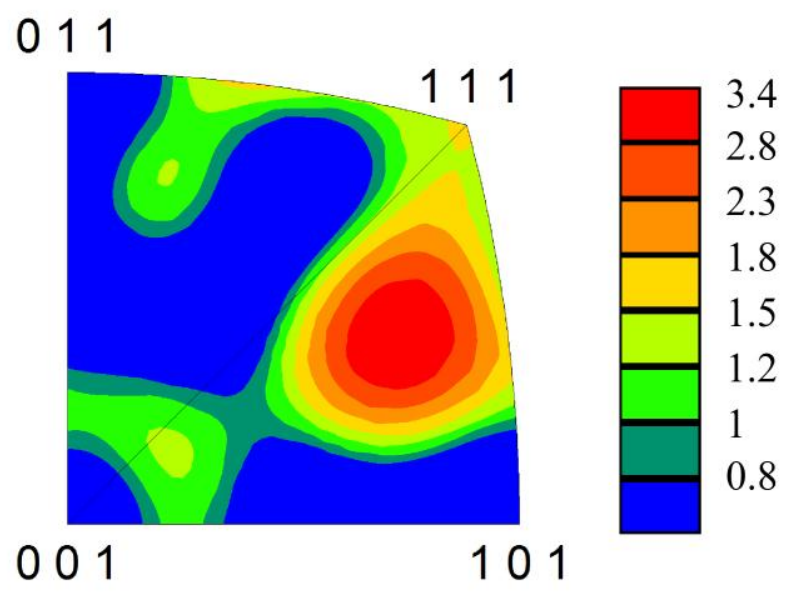




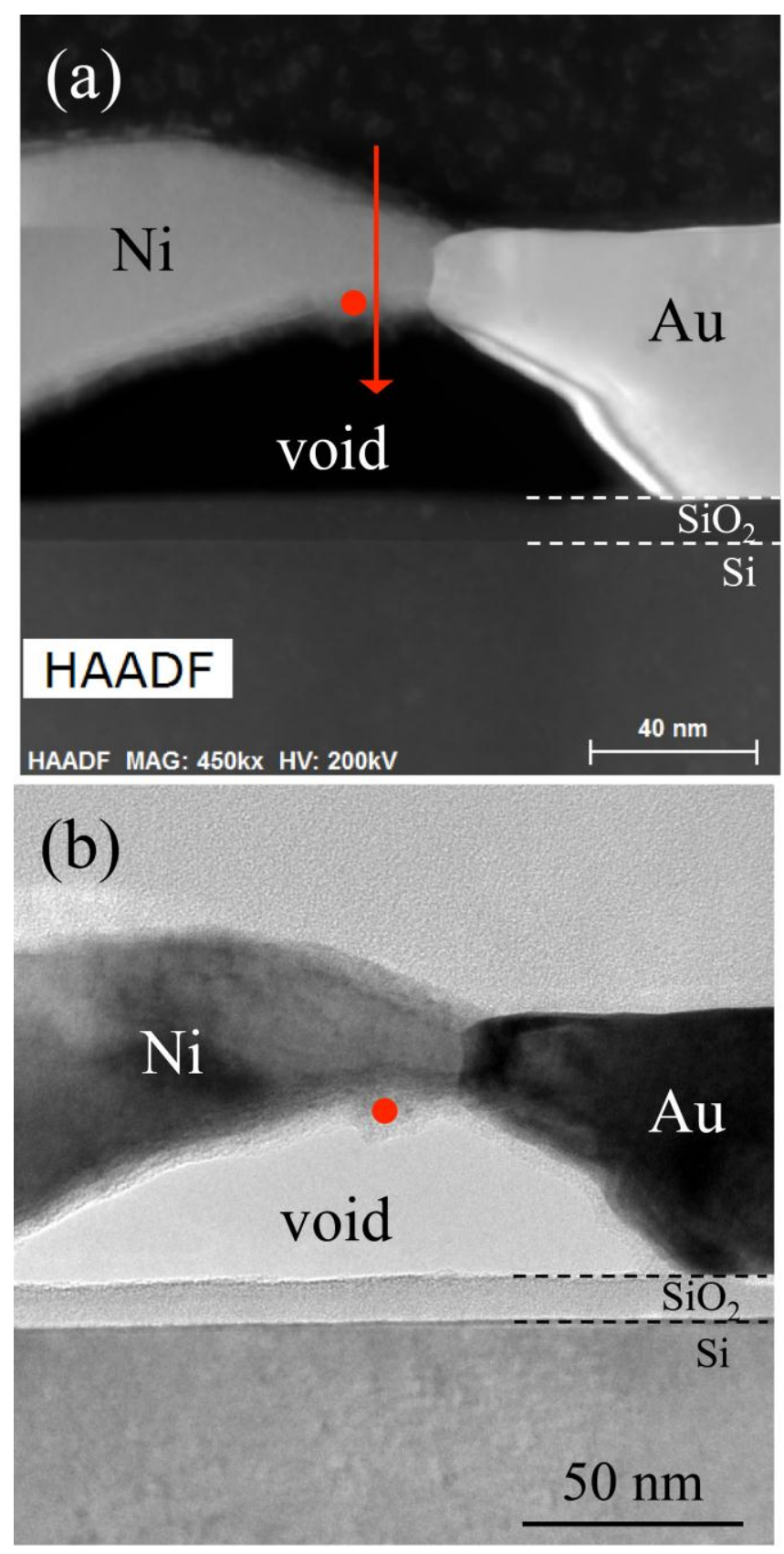



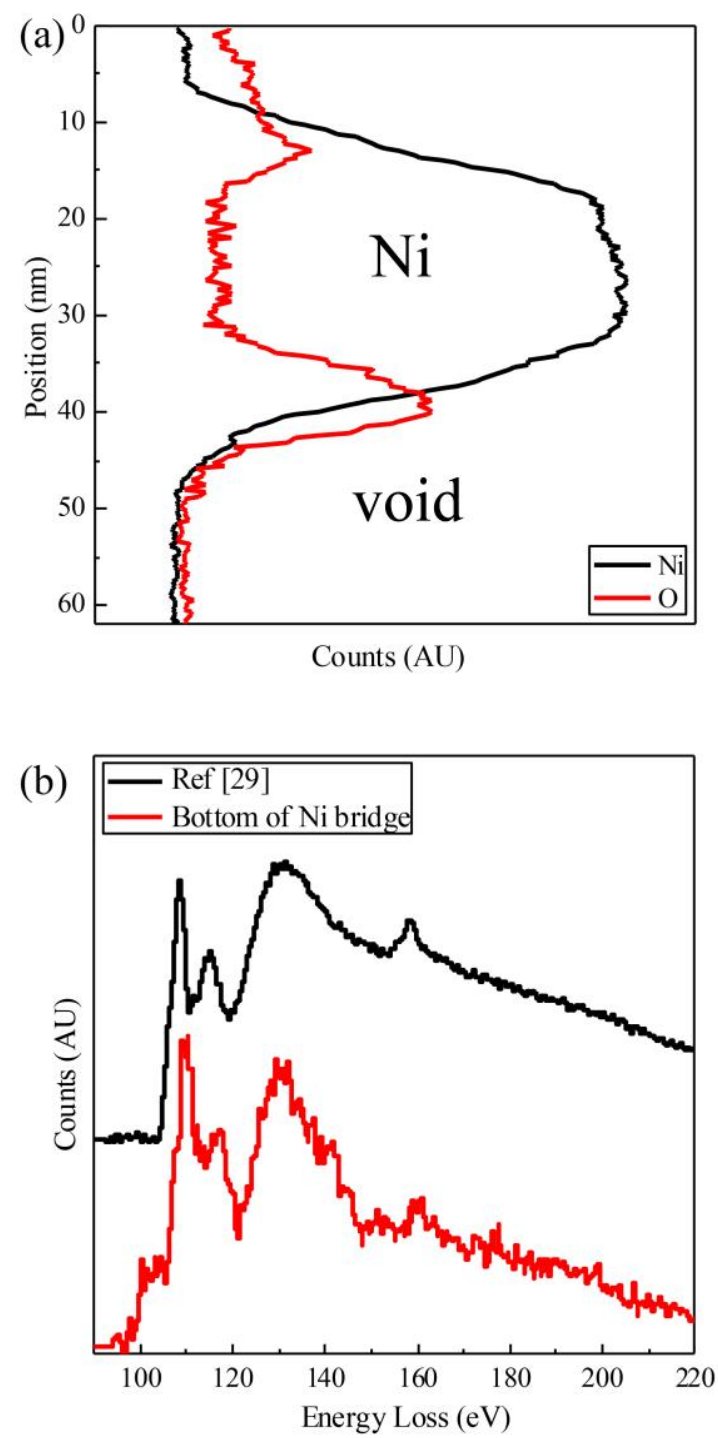


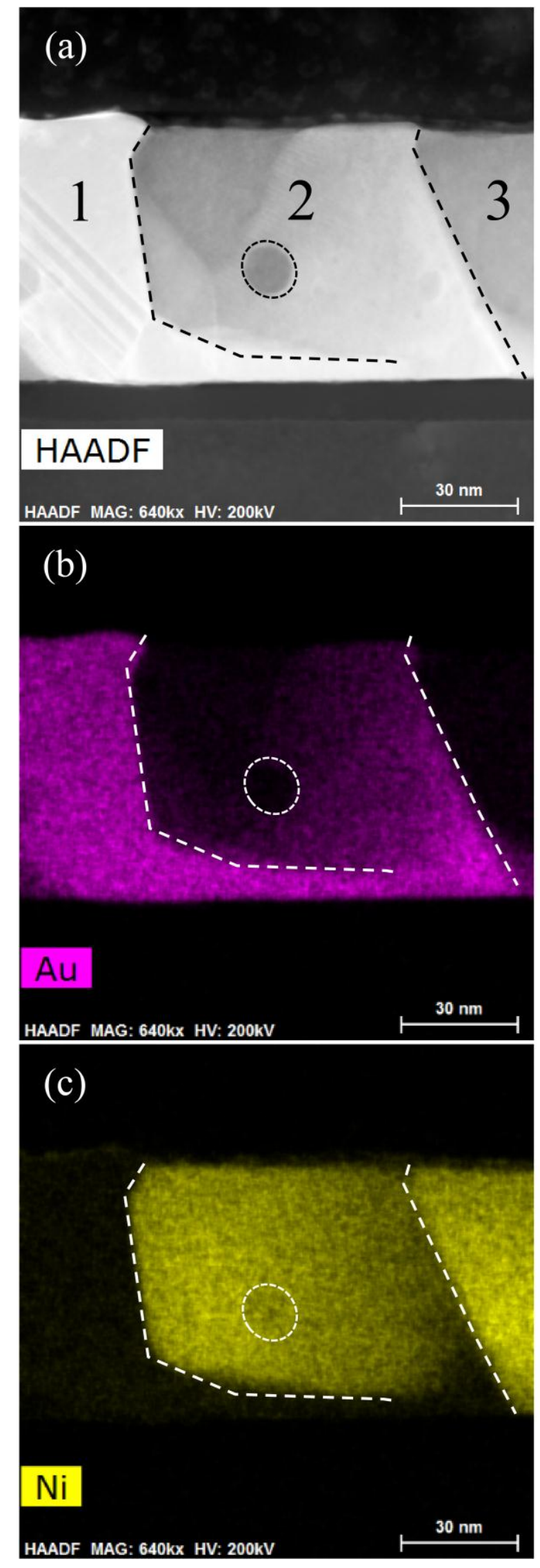




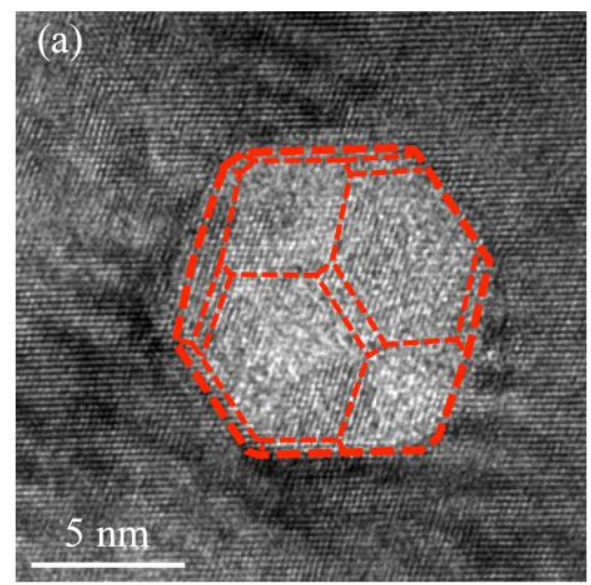

(b)

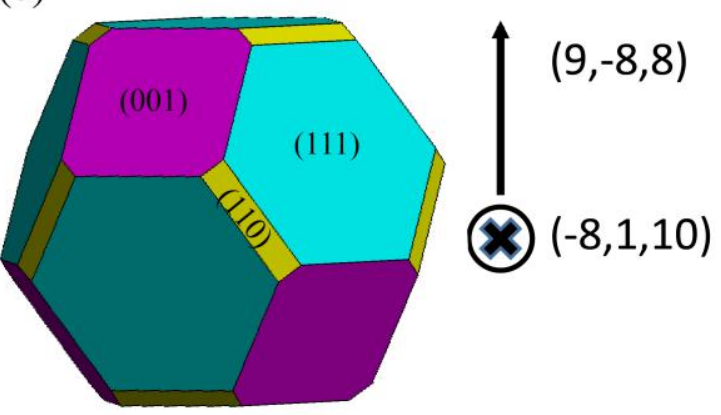




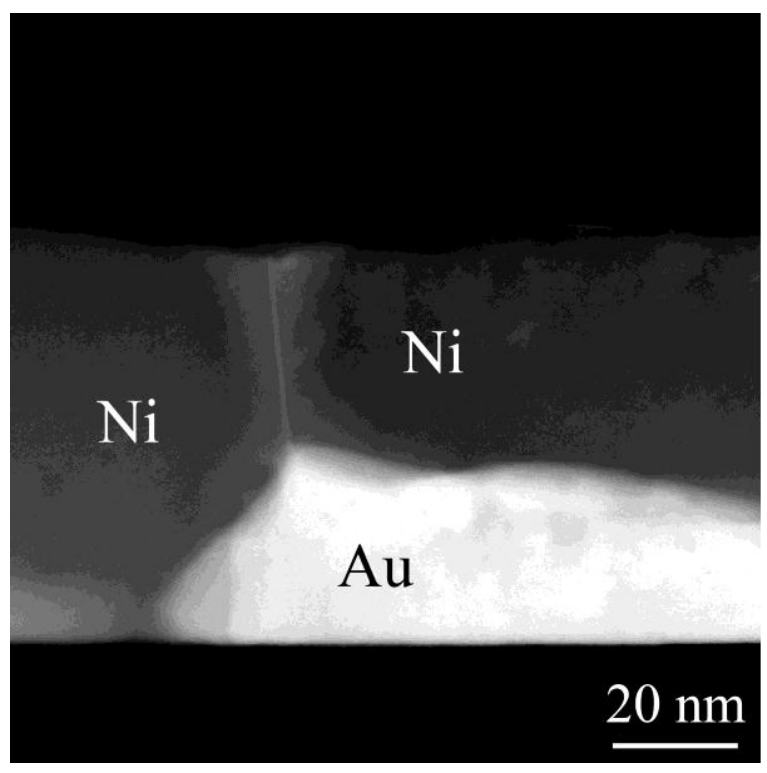

